\title{
The earthworm - Verminephrobacter symbiosis: an emerging experimental system to study extracellular symbiosis
}

\author{
Marie B. Lund ${ }^{1}$ *, Kasper U. Kjeldsen ${ }^{2}$ and Andreas Schramm ${ }^{3}$ \\ 1 Aarhus Institute of Advanced Studies, Aarhus University, Aarhus, Denmark \\ 2 Section for Microbiology, Department of Bioscience, Center for Geomicrobiology, Aarhus University, Aarhus, Denmark \\ ${ }^{3}$ Section for Microbiology, Department of Bioscience, Aarhus University, Aarhus, Denmark
}

\section{Edited by:}

Joerg Graf, University of

Connecticut, USA

Reviewed by:

Rita V. M. Rio, West Virginia

University, USA

Seana Kelyn Davidson, University of

Washington, USA

Adam Silver, University of Hartford, USA

${ }^{*}$ Correspondence:

Marie B. Lund, Aarhus Institute of Advanced Studies, Aarhus

University, Høegh-Guldbergs

Gade 6B, 8000 Aarhus, Denmark

e-mail:mblu@aias.au.dk
Almost all Lumbricid earthworms (Oligochaeta: Lumbricidae) harbor extracellular species-specific bacterial symbionts of the genus Verminephrobacter (Betaproteobacteria) in their nephridia. The symbionts have a beneficial effect on host reproduction and likely live on their host's waste products. They are vertically transmitted and presumably associated with earthworms already at the origin of Lumbricidae 62-136 million years ago. The Verminephrobacter genomes carry signs of bottleneck-induced genetic drift, such as accelerated evolutionary rates, low codon usage bias, and extensive genome shuffling, which are characteristic of vertically transmitted intracellular symbionts. However, the Verminephrobacter genomes lack AT bias, size reduction, and pseudogenization, which are also common genomic hallmarks of vertically transmitted, intracellular symbionts. We propose that the opportunity for genetic mixing during part of the host-symbiont life cycle is the key to evade drift-induced genome erosion. Furthermore, we suggest the earthworm-Verminephrobacter association as a new experimental system for investigating host-microbe interactions, and especially for understanding genome evolution of vertically transmitted symbionts in the presence of genetic mixing.

Keywords: host-symbiont interactions, symbiosis, Verminephrobacter, earthworms, genome evolution, nephridia

\section{INTRODUCTION}

The importance of symbiosis in providing hosts with new biological function has long been recognized (Buchner, 1965; Margulis and Fester, 1991). Symbiotic associations display a fascinating complexity and intimacy between the partners, which have been studied in increasing detail in a variety of model systems. Model systems where the symbiotic partners can be cultured and manipulated separately, e.g., the symbiosis between squids and their bioluminescent symbionts, Vibrio fischeri, are highly valuable when examining function, specificity, and host-microbe interactions during initiation or persistence of the symbiosis (Ruby, 2008). In other symbiotic systems, e.g., the vertically transmitted obligate endosymbiotic bacteria in insects, the partners are intimately interdependent and cannot be separated. However, these systems have provided remarkable insights in the genoand phenotypic changes accompanying transition to intracellular life over evolutionary time (Baumann, 2005; Moran et al., 2008).

The beneficial earthworm-Verminephrobacter symbiosis is a promising emerging experimental system for investigating hostmicrobe interactions: first, earthworms are readily collected in the wild and easily maintained in the lab. Second, the symbiotic partners can be cultured independently (Davidson and Stahl, 2006; Lund et al., 2010b) and the symbiont is genetically tractable (Dulla et al., 2012) allowing important aspects of specificity and initiation of the symbiosis to be explored. Genome sequencing of two Verminephrobacter isolates (Pinel, 2009; Kjeldsen et al., 2012) has revealed that in spite of their ancient association, host fidelity, and strict vertical transmission the symbiont genomes are not reduced in size or eroded as commonly seen in heritable insect endosymbionts (Moran et al., 2009; Toft and Andersson, 2010), but instead follow a different evolutionary path. This mini-review summarizes the collective findings on symbiont diversity, transmission, function, and genome evolution in the earthworm-Verminephrobacter symbiosis. Finally, we propose the earthworm-Verminephrobacter association as a new experimental system for genome evolution of vertically transmitted symbionts in the presence of genetic mixing.

\section{EARTHWORM NEPHRIDIAL SYMBIONTS}

The symbiotic bacteria of the earthworm nephridia (excretory organs) were first discovered through microscopy studies performed by Knop in 1926. The rod-shaped bacteria are confined to the ampulla, a specific region of the nephridia, where they form a dense biofilm (Knop, 1926; Pandazis, 1931; Schramm et al., 2003). The nephridia are found in pairs in each segment of the worm and consist of a long coiled tube leading from the opening to the coelomic cavity, through three major loops, finally exiting the body wall via an exterior pore (Figure 1A). The passing of fluids from the coelom to the exterior plays an important role in both osmoregulation and excretion of nitrogenous waste (Laverack, 1963). 

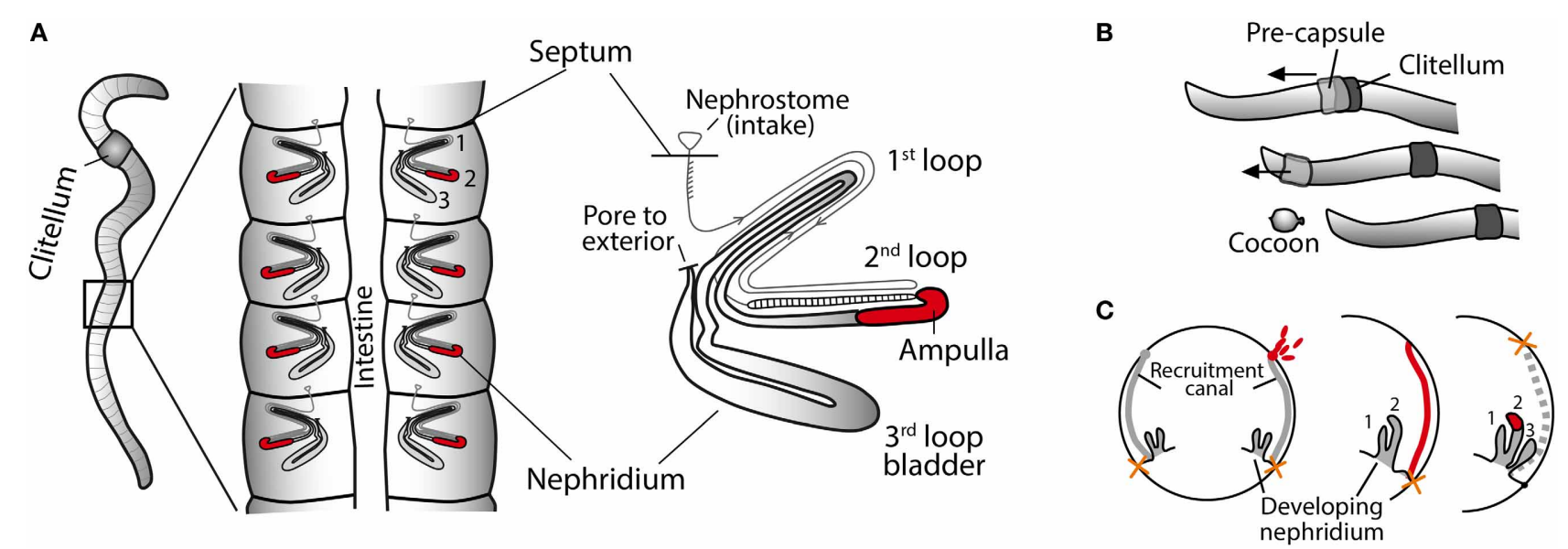

FIGURE 1 | Earthworm anatomy and reproduction. (A) Schematic outline of nephridia in an earthworm. Middle diagram: dissected earthworm with a pair of nephridia attached to the body wall in each segment. Right diagram: detail of single nephridium showing the three major loops. The symbionts are restricted to the ampulla (red). (Modified from Schramm et al., 2003). (B) Cocoons are produced as a secretion of a slime tube (pre-capsule) from the clitellum. Sperm, eggs, and symbionts are deposited in the pre-capsule as the worm crawls backwards out of it. Fertilization takes place in the cocoon.
(C) Symbiont colonization of the nephridia during embryo development. Cross section of earthworm embryo at three different developmental stages: (1) the symbionts (red) aggregate at the opening to the recruitment canal, (2) the symbionts (red) migrate into the recruitment canal, (3) the symbionts colonize the nephridia. Finally the nephridiopore breaks through the body wall. It is unknown if the recruitment canal remains or disappears after colonization. After hatching the worms can no longer take up the symbionts. [Panel (C) is adapted from Davidson and Stahl, 2008].
The nephridial bacteria comprise a separate clade named Verminephrobacter (Schramm et al., 2003; Pinel et al., 2008) within the Betaproteobacteria and their specific localization in the nephridia was confirmed by fluorescence in situ hybridization (FISH) (Schramm et al., 2003; Lund et al., 2010a). The Verminephrobacter symbionts are species-specific and occur almost universally in lumbricid earthworms being consistently present in 28 out of 35 investigated species (Lund et al., 2010a; Davidson et al., 2013). In addition to the Verminephrobacter symbionts, most earthworms harbor a mixed population of nephridial bacteria predominantly belonging to a few groups; Flexibacter-affiliated bacteria are found in about half of the species and other more sporadically occurring bacterial types include; Ochrobactrum and the common soil bacteria Herbaspirillum, Azospirillum, Microbacteriaceae sp., and Variovorax (Davidson et al., 2013). Only eight of the 35 investigated lumbricid earthworm species are exclusively colonized by Verminephrobacter (Lund et al., 2010a; Davidson et al., 2013). When Verminephrobacter is present in a mixed nephridial community they have been observed to form a biofilm attached to the lumen wall, whereas the other bacteria occupy the lumen (Davidson et al., 2010; Lund, unpublished). Other earthworm families (nine out of 11 investigated) within the Crassiclitellata also harbor diverse (non-Verminephrobacter) bacteria in their nephridia (Davidson et al., 2013) as do leeches, a sister group of oligochaetes (Wenning et al., 1993; Graf et al., 2006; Kikuchi et al., 2009). The significance of these nephridial bacteria is unknown.

\section{VERTICAL TRANSMISSION OF VERMINEPHROBACTER SYMBIONTS}

The Verminephrobacter symbionts are transmitted vertically, i.e., passed on directly from parents to offspring, as shown for the common brandling worm, Eisenia fetida (Davidson and Stahl, 2006). Earthworms are hermaphrodites, and during mating the worms cross-fertilize by exchanging spermatozoa, which are stored in sperm sacs for days to months until cocoon production is initiated (Butt and Nuutinen, 1998). Cocoons are formed with the secretion of a tube-shaped precapsule from the clitellum. The worm deposits albumin, egg cells and the stored spermatozoa in the precapsule as it crawls backwards out of the capsule (Figure 1B). Fertilization takes place in the cocoon (Sims and Gerard, 1985; Edwards and Bohlen, 1996). The Verminephrobacter symbionts are also deposited in the precapsule, presumably by shedding through the nephridiopore. During embryonic development, the symbionts colonize the nephridia and when the hatchlings leave the cocoons, they are fully colonized. After hatching, the nephridia can no longer be colonized by new symbionts (Davidson and Stahl, 2006). The hermaphroditic earthworms can mate and store sperm from multiple partners (Porto et al., 2012) and symbionts could potentially also be exchanged during mating. Such hypothetical multi-parental symbiont transmission would hugely increase the scope for genetic mixing in the symbiont population.

During embryonic development the nephridia develop first internally, and the nephridiopore (the opening to the external side) is the last to develop (Knop, 1926). The Verminephrobacter symbionts do not colonize via the nephridiopore, as one might expect, but rather through a canal-like structure (recruitment canal) that forms during nephridia development (Figure 1C); the bacteria aggregate at the opening of the recruitment canal and migrate inside, where they persist until the nephridium is sufficiently developed to allow the final colonization of the ampulla (Davidson and Stahl, 2008). The nephridiopore forms after colonization and the recruitment canal, which may just be 
a transient developmental structure, is closed for further colonization (Davidson and Stahl, 2008). Site-directed mutagenesis of motility genes in Verminephrobacter eiseniae, EF05-2r, has shown that both flagellar and twitching motility is involved in colonization of the developing embryo (Dulla et al., 2012). Flagellar mutants (both $f \lg K$ and $f \lg L$ ) can colonize the bladder but do not migrate into the ampulla and they are eventually lost from juvenile worms. Type IV pili mutants (pilB and pilC double mutants) are incapable of colonizing the embryos (Dulla et al., 2012). Thus, pili structures are necessary for adhesion or migrating through the recruitment canal and flagellar motility is necessary for the final migration to the ampulla. The importance of motility or adhesion for symbiosis initiation has also been demonstrated in the nodulating bacterium Rhizobium meliloti (Ames and Bergman, 1981; Malek, 1992) and in Vibrio fischeri symbionts of squids (Graf et al., 1994) where pili have an important role in host colonization (Stabb and Ruby, 2003).

\section{BENEFICIAL EFFECT OF VERMINEPHROBACTER SYMBIONTS ON HOST REPRODUCTION}

The function of the symbiosis is still a conundrum; aposymbiotic worms can be reared in the lab by submerging newly laid cocoons in antibiotics and these worms can produce viable, aposymbiotic offspring (Davidson et al., 2010; Lund et al., 2010b). A controlled study of life-history traits of symbiotic and aposymbiotic Aporrectodea tuberculata worms receiving either a nutrient rich food source (cow dung) or a nutrient poor food source (straw) showed both a delay in sexual maturity (by approximately 3 weeks) and reduced cocoon hatching success (25 vs. $57 \%$ ) for aposymbiotic compared to symbiotic worms when they received the low-nutrient diet (Lund et al., 2010b). Worms receiving the high-nutrient diet had a higher cocoon hatching success of 84 and $79 \%$ for symbiotic and aposymbiotic worms, respectively. This indicates that well-fed worms can deliver more nutrients to their cocoons and that the presence of symbionts can partly compensate for the nutrient deficiency. No difference in amino acid content was found in cocoons from symbiotic and aposymbiotic worms (Lund et al., 2010b). Instead, the symbionts may provide vitamins or other essential co-factors for which biosynthesis pathways [e.g., for pyrroloquinoline quinone (PQQ) and riboflavin] have been identified in the genome of $V$. eiseniae (Pinel, 2009) and V. aporrectodeae ssp. tuberculatae (AFAL00000000). Alternatively, the symbionts could protect the developing embryos from pathogens; potential antimicrobial properties of Verminephrobacter sp. are yet to be investigated.

\section{EVOLUTION OF VERMINEPHROBACTER SYMBIONTS ANCIENT SYMBIOTIC RELATIONSHIP}

Vertically transmitted symbionts commonly co-diversify with their hosts, as shown for a wide range of insect primary endosymbionts (Chen et al., 1999; Clark et al., 2000; Baumann, 2005; Hosokawa et al., 2006; Takiya et al., 2006), chemoautotrophic symbionts in marine animals (Peek et al., 1998), and symbionts of termite gut protists (Noda et al., 2007). Co-diversification was also detected between 23 earthworms species and their Verminephrobacter symbionts (Lund et al., 2010a), albeit only clearly resolvable in four clades, due to poor resolution of host phylogeny. Therefore, the symbiosis likely originate in the last common ancestor of Lumbricid earthworms (62-136 million years ago Bouché, 1983; Lund et al., 2010a; Davidson et al., 2013). One study of Lumbricus terrestris and its Verminephrobacter symbionts concluded that the partners do not co-disperse, and thus cannot co-diversify (Bakke et al., 2011). However, only nine individuals (five from Canada, three from Germany, and one from Norway) were included in the analysis and no statistical support was shown for the phylogenetic trees. With such a low number of individuals it is difficult to distinguish if the sequence diversity is due to random variation or if there is a true biogeographic signal.

\section{ACCELERATED EVOLUTIONARY RATES}

Obligate intracellular symbionts live in genetic isolation from other microbes and experience periodic population bottlenecks during vertical transmission from one host generation to the next (Moran and Plague, 2004; Bright and Bulgheresi, 2010). With heritable symbionts of insects as prime examples, this lifestyle has profound effects on the genome evolution of these organisms. For example, bottleneck-induced genetic drift causes genome erosion manifesting as accelerated substitution rates, pseudogenization, genome-wide biased base composition, gene loss, and overall genome miniaturization (Moran et al., 2008; Moya et al., 2008; Toft and Andersson, 2010).

In contrast, very little (and conflicting) information is available about the evolution of extracellular vertically transmitted symbionts, which have a greater potential for genetic mixing than their intracellular counter parts. For example, stinkbug symbionts have the same signatures of reductive genome evolution as intracellular insect symbionts (Hosokawa et al., 2006; Kikuchi et al., 2009), while conversely, the extracellular endosymbionts of gutless oligochaetes have the same genome size and base composition as their free-living relatives (Woyke et al., 2006). The stinkbug symbionts reside in specialized isolated crypts (Kikuchi et al., 2009), which may restrict the symbionts from genetic exchange with other microbes, hence effectively resembling an intracellular genetic isolation. For the symbionts of gutless oligochaetes, the mode of transmission is still uncertain (Woyke et al., 2006) and the microbes may experience free-living life stages in marine sediments, thereby evading population bottlenecks entirely.

The fully sequenced genome of V. eiseniae, the nephridial symbiont of Eisenia fetida, has a size of $5.6 \mathrm{Mb}$ and a GC content of $65.3 \%$ (Pinel, 2009), and thereby does not show any signs of genome reduction or AT-bias, in spite of the symbiont host fidelity, vertical transmission, and the high age of the symbiosis (62-136 million years (MY)). However, compared to free-living close relatives, the Verminephrobacter symbionts exhibit accelerated evolutionary rates in the RNA polymerase subunit B gene $(r p o B)$ (Lund, 2009). And when calibrating Verminephrobacter $16 \mathrm{~S}$ rRNA substitution rates with the age of the symbiosis, the rate (0.0117-0.0257 substitution per site per 50 MY Lund et al., 2010a) is comparable to rate estimates for older endosymbiotic associations; e.g., the 160-280 MY old symbiosis between Buchnera and aphids (0.0075-0.0232 substitutions per site per 50 MY Moran et al., 1993) and the 135-180 MY old endosymbiosis in woodroaches $(0.0084-0.0111$ substitutions per site per 50 MY Maekawa et al., 2005). These accelerated evolutionary rates 
indicate that the Verminephrobacter symbionts are indeed affected by bottleneck-induced genetic drift.

Genome-wide evidence for accelerated evolutionary rates in the Verminephrobacter genus is also found in a four-genome comparison of the two available symbiont genomes with the genomes of two closely related Acidovorax species (Kjeldsen et al., 2012). In the 876 orthologous genes used in the analysis the ratio of nonsynonymous to synonymous substitutions $(\mathrm{dN} / \mathrm{dS})$ was on average $50 \%$ higher in the symbionts than in the free-living relatives although the genes were overall evolving under strong purifying selection (average $\mathrm{dN} / \mathrm{dS}=0.09 \pm 0.07$ Kjeldsen et al., 2012) as is the case for other heritable symbiont genomes (Moran et al., 2009). The Verminephrobacter symbionts also had less codon usage bias (Kjeldsen et al., 2012), which is another clear indicator of relaxed purifying selection in the symbiont lineage. At the same time Verminephrobacter also showed signs of adaptive evolution in 89 genes evolving under positive selection compared to only 7 positively selected genes in the free-living lineages (Kjeldsen et al., 2012). The positively selected genes in Verminephrobacter may play key roles in the function of the symbiosis.

\section{A GENOME IN FLUX}

Interestingly, the genome of $V$. eiseniae has no synteny to the genomes of two closely related Acidovorax species (Pinel, 2009), or the large contigs of the partially sequenced symbiont of the earthworm A. tuberculata (Figure S1), which indicates that the Verminephrobacter genomes are actively rearranging. This is supported by the very low DNA-DNA hybridization values found between three strains of Verminephrobacter symbionts from different earthworm hosts (28.3-58.8\%) (Lund et al., 2011). V. eiseniae also contains a high number of palindromic repeats and insertion sequence elements; about $2.3 \%$ of its genome is comprised of one type of palindromic repeat, VeiPR1, unique to $V$. eiseniae (Pinel, 2009). A high load of mobile DNA is expected in organisms that have recently transitioned to an obligate intracellular lifestyle (Moran and Plague, 2004), as bottleneck-induced genetic drift will allow mobile genetic elements to proliferate in the genome. However, with time, mobile DNA elements will be inactivated and lost, and new mobile elements can only be acquired through recombination with other organisms (Figure 2B). This explains the almost total absence of mobile DNA in old obligate insect endosymbionts and the high number of mobile elements in more recent obligate host associates (Moran and Plague, 2004; Bordenstein and Reznikoff, 2005; Moran et al., 2009). According to this theory the earthwormVerminephrobacter symbiosis resembles a young symbiosis in transition toward genome reduction; however, low pseudogenization (Pinel, 2009), overall strong purifying selection (Kjeldsen

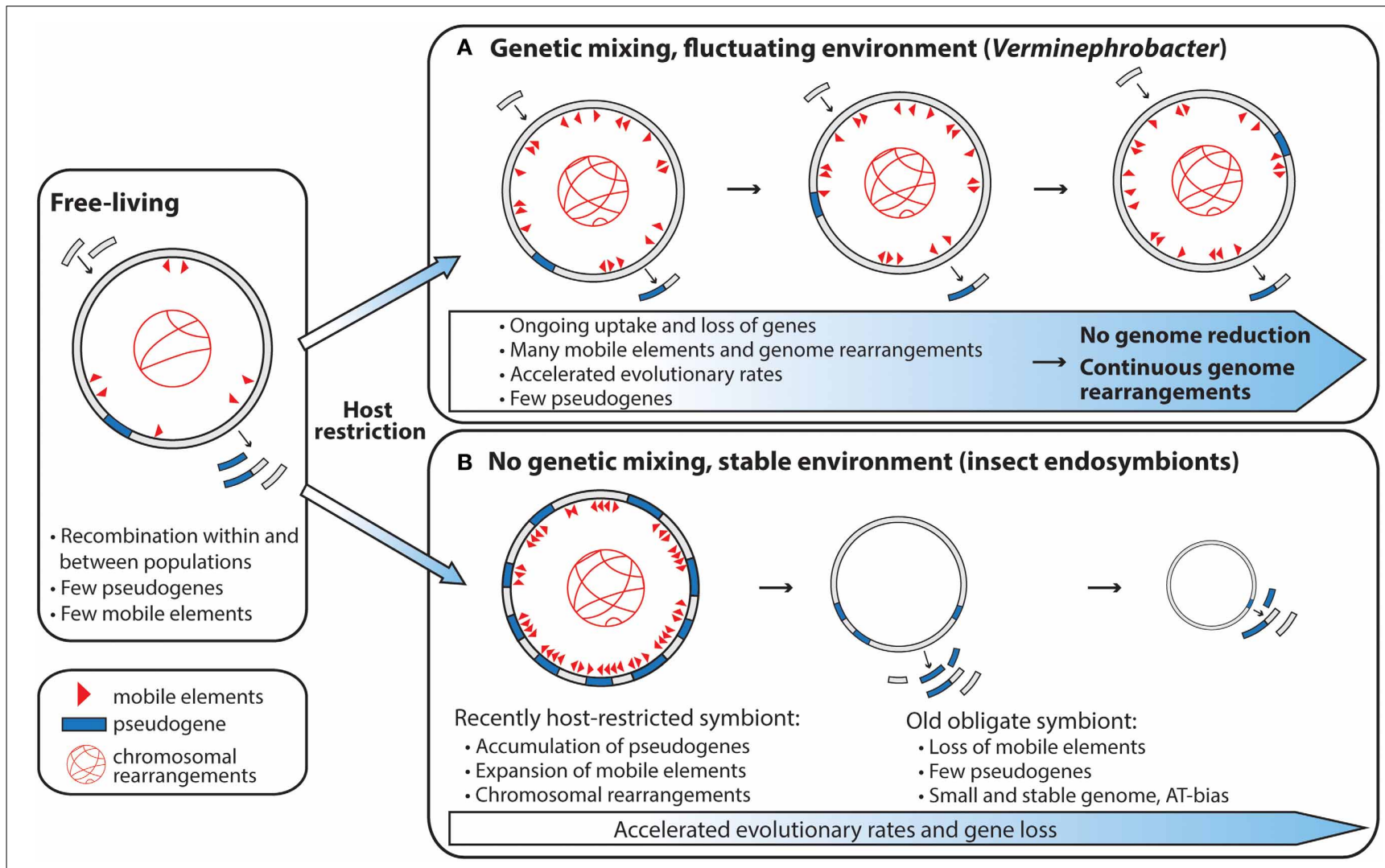

FIGURE 2 | Genome evolution of vertically transmitted symbionts after host restriction. The scope for genetic mixing has large implications for symbiont genome evolution. (A) Genome evolution of vertically transmitted symbionts experiencing environmental fluctuations during the host-symbiont life cycle and with the opportunity for genetic mixing. (B) Genome evolution of vertically transmitted symbionts living in genetic isolation (Panel (B) redrawn from McCutcheon and Moran, 2012). 
et al., 2012), and a high age of the symbiosis (62-136 MY, Bouché, 1983; Lund et al., 2010a) do not support this theory.

\section{GENOME EVOLUTION OF A VERTICALLY TRANSMITTED, EXTRACELLULAR SYMBIONT}

Although the Verminephrobacter symbionts have high host fidelity and are vertically transmitted, their extracellular lifestyle, and thereby scope for genetic mixing, leads to a different pattern of genome evolution compared to intracellular symbionts. Unlike obligate intracellular symbionts that live in genetically isolated, stable environments, the Verminephrobacter are subjected to two ecologically different environments; the nephridia and the cocoon. In the cocoon they encounter a mixed community of soil bacteria (Zachmann and Molina, 1993; Davidson et al., 2010) and, as discussed above, some of these co-colonize the nephridia together with Verminephrobacter in many earthworm species (Davidson and Stahl, 2008; Lund et al., 2010a; Davidson et al., 2013). The environmental fluctuations throughout the host-symbiont life cycle may select against the loss of genes required to survive in more diverse environments, and the opportunity for genetic exchange with other microorganisms can counteract deleterious effects of bottleneck-induced genetic drift (Figure 2A). In addition, the hosts could potentially exchange symbionts during mating which can lead to multi-parental inheritance. Multi-parental inheritance will enable homologous recombination between otherwise separate symbiont lineages and thus further counteract bottleneck-induced genetic drift. The importance of homologous recombination within the symbiont population has not yet been investigated in the earthworm-Verminephrobacter system or, to our knowledge, in any other symbiotic system.

The host-symbiont life cycle and degree of genetic isolation has huge implications for the genome evolution of vertically transmitted symbionts (Bright and Bulgheresi, 2010) and we propose that the scope for genetic mixing is important in offering the symbiont an escape from the deleterious effect of bottleneck-induced genetic drift. The degree of genetic exchange within the earthworm symbiont populations and with other microbes encountered in the cocoons or nephridia remains to be investigated. However, the ability to cultivate the symbionts makes it possible to investigate recombination using multi locus sequence typing (MLST) and whole genome comparisons. The earthworm-Verminephrobacter symbiosis is, to our knowledge, the only experimental system in which the genome evolution of vertically transmitted, host specific, extracellular symbionts has been investigated. We predict that other vertically transmitted symbionts experiencing genetic mixing will evade genome erosion in a manner similar to the Verminephrobacter symbionts; this theoretical framework remains to be tested in other symbiotic systems.

\section{ACKNOWLEDGMENTS}

The original work of the authors was funded by The Danish Council for Independent Research |Natural Sciences (FNU), the Carlsberg Foundation, Danish National Research Foundation, the EU Marie Curie Initial Training Network "Symbiomics," and the Aarhus Institute of Advanced Studies (AIAS). We thank Britta
Poulsen and Annemarie Højmark for expert help in the laboratory and for keeping the worms happy; Thomas Bataillon, Martin Holmstrup, Ralf Rabus, Flávia Viana, David Stahl, and Nicolas Pinel are acknowledged for fruitful discussions.

\section{SUPPLEMENTARY MATERIAL}

The Supplementary Material for this article can be found online at: http://www.frontiersin.org/journal/10.3389/fmicb.2014. 00128/abstract

Figure S1 | Synteny mapping of 14 major genome contigs of the Verminephrobacter aporrectodeae At $4^{\top}$ draft genome (AFAL00000000) on to the fully sequenced chromosome of Verminephrobacter eiseniae EF01-2 $^{\boldsymbol{\top}}$ (CP000542). Not drawn to scale. The plot is based on translated amino acid alignments constructed with PROmer of the MUMmer package (Kurtz et al., 2004). Only the single longest consistent alignment was kept for any contig region. The plot was drawn using Circos (Krzywinski et al., 2009). Numbers below contig names represent the total length of a contig/the average length of its aligned regions.

\section{REFERENCES}

Ames, P., and Bergman, K. (1981). Competitive advantage provided by bacterial motility in the formation of nodules by Rhizobium meliloti. J. Bacteriol. 148, 728-908.

Bakke, B. B., Wilson, R., and Rudi, K. (2011). Prevalence and dispersal of a facultative bacterial symbiont associated with an endemic metazoan host. Pedobiologia 54, 153-157. doi: 10.1016/j.pedobi.2010.12.005

Baumann, P. (2005). Biology of bacteriocyte-associated endosymbionts of plant sap-sucking insects. Annu. Rev. Microbiol. 59, 155-189. doi: 10.1146/annurev.micro.59.030804.121041

Bordenstein, S. R., and Reznikoff, W. S. (2005). Mobile DNA in obligate intracellular bacteria. Nat. Rev. Micro. 3, 688-699. doi: 10.1038/nrmicro1233

Bouché, M. B. (1983). "The establishment of earthworm communities," in Earthworm Ecology - From Darwin to Vermiculture, ed J. E. Satchell (New York, NY: Chapman and Hall), 431-448.

Bright, M., and Bulgheresi, S. (2010). A complex journey: transmission of microbial symbionts. Nat. Rev. Micro. 8, 218-230. doi: 10.1038/nrmicro2262

Buchner, P. (1965). Endosymbiosis of Animals With Plant Microorganisms. New York, NY: Interscience Publishers, John Wiley and Sons.

Butt, K. R., and Nuutinen. V. (1998). Reproduction of the earthworm Lumbricus terrestris Linné after the first mating. Can. J. Zool. 76, 104-109. doi: 10.1139/z97179

Chen, X., Li, S., and Aksoy, S. (1999). Concordant evolution of a symbiont with its host insect species: molecular phylogeny of genus Glossina and its bacteriomeassociated endosymbiont, Wigglesworthia glossinidia. J. Mol. Evol. 48, 49-58. doi: 10.1007/PL00006444

Clark, M., Moran, N., Baumann, P., and Wernegreen, J. (2000). Cospeciation between bacterial endosymbionts (Buchnera) and a recent radiation of aphids (Uroleucon) and pitfalls of testing for phylogenetic congruence. Evolution 54, 517-525. doi: 10.1554/0014-3820(2000)054\%5B0517:CBBEBA\%5D2.0.CO;2

Davidson, S. K., Powell, R. J., and James, S. (2013). A global survey of the bacteria within earthworm nephridia. Mol. Phylo. Evol. 67, 188-200. doi: 10.1016/j.ympev.2012.12.005

Davidson, S. K., Powell, R. J., and Stahl, D. A. (2010). Transmission of a bacterial consortium in Eisenia fetida egg capsules. Environ. Microbiol. 12, 2277-2288. doi: 10.1111/j.1462-2920.2010.02231.x

Davidson, S. K., and Stahl, D. A. (2006). Transmission of nephridial bacteria of the earthworm Eisenia fetida. Appl. Environ. Microbiol. 72, 769-775. doi: 10.1128/AEM.72.1.769-775.2006

Davidson, S. K., and Stahl, D. A. (2008). Selective recruitment of bacteria during embryogenesis of an earthworm. ISME J. 2, 510-518. doi: 10.1038/ismej.2008.16

Dulla, G. F. J., Go, R. A., Stahl, D. A., and Davidson, S. K. (2012). Verminephrobacter eiseniae type IV pili and flagella are required to colonize earthworm nephridia. ISME J. 6, 1166-1175. doi: 10.1038/ismej.2011.183

Edwards, C. A., and Bohlen, P. J. (1996). Biology and Ecology of Earthworms. London: Chapman \& Hall. 
Graf, J., Dunlap, P. V., and Ruby, E. G. (1994). Effect of transposon-induced motility mutations on colonization of the host light organ by Vibrio fischeri. J. Bacteriol. 176, 6986-6991.

Graf, J., Kikuchi, Y., and Rio, R. V. M. (2006). Leeches and their microbiota: naturally simple symbiosis models. Trends Microbiol. 14, 365-371. doi: 10.1016/j.tim.2006.06.009

Hosokawa, T., Kikuchi, Y., Nikoh, N., Shimada, M., and Fukatsu, T. (2006). Strict host-symbiont cospeciation and reductive genome evolution in insect gut bacteria. PLoS Biol. 4:e337. doi: 10.1371/journal.pbio.0040337

Kikuchi, Y., Bomar, L., and Graf, J. (2009). Stratified bacterial community in the bladder of the medicinal leech, Hirudo verbana. Environ. Microbiol. 11, 2758-2770. doi: 10.1111/j.1462-2920.2009.02004.x

Kjeldsen, K. U., Bataillon, T., Pinel, N., De Mita, S., Lund, M. B., Panitz, F., et al. (2012). Purifying selection and molecular adaptation in the genome of Verminephrobacter, the heritable symbiotic bacteria of earthworms. Gen. Biol. Evol. 4, 307-315. doi: 10.1093/gbe/evs014

Knop, J. (1926). Bakterien und Bakteroiden bei Oligochäten. Z. Morphol. Ökol. Tiere. 6, 588-624. doi: 10.1007/BF00464431

Krzywinski, M., Schein, J., Birol, I., Connors, J., Gascoyne, R., Horsman, D., et al. Marra, M. A. (2009). Circos: an Information Aesthetic for Comparative Genomics. Gen. Res. 19, 1639-1645. doi: 10.1101/gr.092759.109

Kurtz, S., Phillippy, A., Delcher, A. L., Smoot, M., Shumway, M., Antonescu, C., et al. (2004). Versatile and open software for comparing large genomes. Genome Biol. 5:R12. doi: 10.1186/gb-2004-5-2-r12

Laverack, M. S. (1963). The Physiology of Earthworms. New York, NY: Pergamon Press.

Lund, M. B. (2009). Evolution and Function of the Earthworm - Verminephrobacter symbiosis. Ph.D. thesis, Aarhus University, Denmark.

Lund, M. B., Davidson, S. K., Holmstrup, M., James, S., Kjeldsen, K. U., Stahl, D. A., et al. (2010a). Diversity and host specificity of the Verminephrobacterearthworm symbiosis. Environ. Microbiol. 12, 2142-2151. doi: 10.1111/j.14622920.2009.02084.x

Lund, M. B., Holmstrup, M., Lomstein, B. A., Damgaard, C., and Schramm, A. (2010b). Beneficial effect of Verminephrobacter nephridial symbionts on the fitness of the earthworm Aporrectodea tuberculata. Appl. Environ. Microbiol. 76, 4738-4743. doi: 10.1128/AEM.00108-10

Lund, M. B., Schätzle, S., Schramm, A., and Kjeldsen, K. U. (2011). Verminephrobacter aporrectodeae sp. nov. subsp. tuberculatae and subsp. caliginosae, the specific nephridial symbionts of the earthworms Aporrectodea tuberculata and A. caliginosa. Antonie Van Leeuwenhoek. 101, 507-514. doi: 10.1007/s10482-011-9659-X

Maekawa, K., Park, Y. C., and Lo, N. (2005). Phylogeny of endosymbiont bacteria harbored by the woodroach Cryptocercus spp. (Cryptocercidae: Blattaria): molecular clock evidence for a late Cretaceous - early Tertiary split of Asian and American lineages. Mol. Phylo. Evol. 36, 728-733. doi: 10.1016/j.ympev.2005.03.033

Malek, W. (1992). The role of motility in the efficiency of nodulation by Rhizobium meliloti. Arch. Microbiol. 158, 26-28. doi: 10.1007/BF00249061

Margulis, L., and Fester, R. (1991). Symbiosis as a Source of Evolutionary Innovation: Speciation and Morphogenesis. Cambridge, MA: Massachusetts institute of technology.

McCutcheon, J. P., and Moran, N. A. (2012). Extreme genome reduction in symbiotic bacteria. Nat. Rev. Microbiol. 10, 13-26. doi: 10.1038/nrmicro2670

Moran, N. A., McLaughlin, H. J., and Sorek, R. (2009). The dynamics and time scale of ongoing genomic erosion in symbiotic bacteria. Science 323, 379-382. doi: $10.1126 /$ science. 1167140

Moran, N. A., Munson, M. A., Baumann, P., and Ishikawa, H. (1993). A molecular clock in endosymbiotic bacteria is calibrated using the insect hosts. Proc. Biol. Sci. 253, 167-171. doi: 10.1098/rspb.1993.0098

Moran, N., McCutcheon, J., and Nakabachi, A. (2008). Genomics and evolution of heritable bacterial symbionts. Annu. Rev. Genet. 42, 165-190. doi 10.1146/annurev.genet.41.110306.130119

Moran, N., and Plague, G. (2004). Genomic changes following host restriction in bacteria. Curr. Opin. Genet. Dev. 14, 627-633. doi: 10.1016/j.gde.2004.09.003
Moya, A., Peretó, J., Gil, R., and Latorre, A. (2008). Learning how to live together: genomic insights into prokaryote-animal symbioses. Nat. Rev. Genet. 9, 218-229. doi: 10.1038/nrg2319

Noda, S., Kitade, O., Inoue, T., Kawai, M., Kanuka, M., Hiroshima, K. et al. (2007). Cospeciation in the triplex symbiosis of termite gut protists (Pseudotrichonympha spp.), their hosts, and their bacterial endosymbionts. Mol. Ecol. 16, 1257-1266. doi: 10.1111/j.1365-294X.2006.03219.x

Pandazis, G. (1931). Zur Frage der Bakteriensymbiose bei Oligochäten. Zentlbl. Bakteriol: Parasitenkd. Infekt. 120, 440-453.

Peek, A. S., Feldman, R. A., Lutz, R. A., and Vrijenhoek, R. C. (1998). Cospeciation of chemoautotrophic bacteria and deep sea clams. Proc. Natl. Acad. Sci. U.S.A. 95, 9962-9966. doi: 10.1073/pnas.95.17.9962

Pinel, N. (2009). Physiological and Genomic Insights into the Biology of Verminephrobacter eiseniae, a Bacterial Symbiont of the Earthworm Eisenia fetida. Ph.D. thesis, University of Washington, Seattle, Washington, USA.

Pinel, N., Davidson, S. K., and Stahl, D. A. (2008). Verminephrobacter eiseniae gen. nov., sp. nov., a nephridial symbiont of the earthworm Eisenia foetida (Savigny). Int. J. Syst. Evol. Microbiol. 58, 2147-2157. doi: 10.1099/ijs.0.65174-0

Porto, P. G., Velando, A., and Domínguez, J. (2012). Multiple mating increases cocoon hatching success in the earthworm Eisenia andrei (Oligochaeta: Lumbricidae). Biol. J. Linnean. Soc. 107, 175-181. doi: 10.1111/j.10958312.2012.01913.x

Ruby, E. G. (2008). Symbiotic conversations are revealed under genetic interrogation. Nat. Rev. Micro. 6, 752-762. doi: 10.1038/nrmicro1958

Schramm, A., Davidson, S. K., Dodsworth, J. A., Drake, H. L., Stahl, D. A., and Dubilier, N. (2003). Acidovorax-like symbionts in the nephridia of earthworms. Environ. Microbiol. 5, 804-809. doi: 10.1046/j.1462-2920.2003.00474.x

Sims, R. W., and Gerard, B. M. (1985). Earthworms. Leiden: J. Brill/Dr. W. Backhuys.

Stabb, E. V., and Ruby, E. G. (2003). Contribution of pilA to competitive colonization of the squid Euprymna scolopes by Vibrio fischeri. Appl. Environ. Microbiol. 69, 820-826. doi: 10.1128/AEM.69.2.820-826.2003

Takiya, D. M., Tran, P. L., Dietrich, C. H., and Moran, N. A. (2006). Co-cladogenesis spanning three phyla: leafhoppers (Insecta: Hemiptera: Cicadellidae) and their dual bacterial symbionts. Mol. Ecol. 15, 4175-4191. doi: 10.1111/j.1365294X.2006.03071.x

Toft, C., and Andersson, S. G. E. (2010). Evolutionary microbial genomics: insights into bacterial host adaptation. Nat. Rev. Genet. 11, 465-475. doi: $10.1038 / \operatorname{nrg} 2798$

Wenning, A., Cahill, M., and Greisinger, U. (1993). Organogenesis in the leech: development of nephridia, bladders and their innervation. Roux's Arch. Dev Biol. 202, 329-340. doi: 10.1007/BF00188732

Woyke, T., Teeling, H., Ivanova, N. N., Huntemann, M., Richter, M., Gloeckner, F. O., et al. (2006). Symbiosis insights through metagenomic analysis of a microbial consortium. Nature 443, 950-955. doi: 10.1038/nature05192

Zachmann, J., and Molina, J. (1993). Presence of culturable bacteria in cocoons of the earthworm Eisenia fetida. Appl. Environ. Microbiol. 59, 1904-1910.

Conflict of Interest Statement: The authors declare that the research was conducted in the absence of any commercial or financial relationships that could be construed as a potential conflict of interest.

Received: 02 January 2014; accepted: 13 March 2014; published online: 28 March 2014. Citation: Lund MB, Kjeldsen KU and Schramm A (2014) The earthwormVerminephrobacter symbiosis: an emerging experimental system to study extracellular symbiosis. Front. Microbiol. 5:128. doi: 10.3389/fmicb.2014.00128

This article was submitted to Microbial Symbioses, a section of the journal Frontiers in Microbiology.

Copyright (c) 2014 Lund, Kjeldsen and Schramm. This is an open-access article distributed under the terms of the Creative Commons Attribution License (CC BY). The use, distribution or reproduction in other forums is permitted, provided the original author(s) or licensor are credited and that the original publication in this journal is cited, in accordance with accepted academic practice. No use, distribution or reproduction is permitted which does not comply with these terms. 